Author affiliations and support information (if applicable) appear at the end of this article.

Published at jco.org on November 8, 2017

Clinical trial information: NCT02054806.

Corresponding author: Toshihiko Doi, MD, $\mathrm{PhD}$, Department of Gastrointestinal Oncology, National Cancer Center East, 6-5-1, Kashiwanoha, Kashiwa, Chiba, 277-8577, Japan; e-mail: tdoi@east. ncc.go.jp.

(c) 2017 by American Society of Clinical Oncology

0732-183X/18/3601w-61w/\$20.00

\title{
Safety and Antitumor Activity of the Anti-Programmed Death-1 Antibody Pembrolizumab in Patients With Advanced Esophageal Carcinoma
}

Toshihiko Doi, Sarina A. Piha-Paul, Shadia I. Jalal, Sanatan Saraf, Jared Lunceford, Minori Koshiji, and Jaafar Bennouna

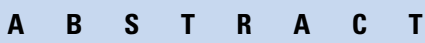

\section{Purpose}

The anti-programmed death-1 antibody pembrolizumab was evaluated in KEYNOTE-028, a multicohort, phase IB study of patients with programmed death ligand-1 (PD-L1)-positive advanced solid tumors. Results from the esophageal carcinoma cohort are reported herein.

\section{Patients and Methods}

Eligible patients with squamous cell carcinoma or adenocarcinoma of the esophagus or gastroesophageal junction in whom standard therapy failed and who had PD-L1-positive tumors received pembrolizumab $10 \mathrm{mg} / \mathrm{kg}$ every 2 weeks for up to 2 years or until confirmed disease progression or intolerable toxicity. Response was assessed every 8 weeks up to 6 months and every 12 weeks thereafter. Primary end points were safety and overall response rate, determined by investigator review per Response Evaluation Criteria in Solid Tumors (version 1.1).

\section{Results}

Among 83 patients with esophageal carcinoma and samples evaluable for PD-L1 expression, 37 (45\%) had PD-L1-positive tumors, and 23 were enrolled. Median age was 65 years; 78\% had squamous histology; and $87 \%$ received $\geq$ two prior therapies for advanced/metastatic disease. As of the data cutoff (February 20, 2017), median follow-up was 7 months (range, 1 to 33 months). Nine patients (39\%) experienced treatment-related adverse events, most commonly decreased appetite, decreased lymphocyte count, generalized rash, and rash (two patients [9\%] each). No grade 4 adverse events or deaths were attributed to pembrolizumab. Overall response rate was $30 \%(95 \% \mathrm{Cl}, 13 \%$ to $53 \%)$; median duration of response was 15 months (range, 6 to 26 months). A six-gene interferon- $\gamma$ gene expression signature analysis suggested that delayed progression and increased response occur among pembrolizumabtreated patients with higher interferon- $\gamma$ composite scores.

\section{Conclusion}

Pembrolizumab demonstrated manageable toxicity and durable antitumor activity in patients with heavily pretreated, PD-L1-positive advanced esophageal carcinoma.

\section{J Clin Oncol 36:61-67. (C) 2017 by American Society of Clinical Oncology}

\section{INTRODUCTION}

Esophageal carcinoma is the eighth most common cancer and the sixth leading cause of cancer-related deaths worldwide, with an estimated 400,000 deaths annually. ${ }^{1,2}$ It is endemic in developing countries, where it is the fourth leading cause of cancer-related deaths. ${ }^{1,2}$ Prevalence of esophageal carcinoma is higher in men than in women, ${ }^{1,2}$ and the incidence and histologic subtype vary based on geographic location. ${ }^{3}$ In the Western Hemisphere, the incidence of adenocarcinoma has increased over the past 25 years, mainly in association with increasing rates of obesity, gastroesophageal reflux disease, and Barrett's esophagus. ${ }^{3}$ In contrast, in other parts of the world, particularly in the esophageal cancer belt, which extends from eastern Turkey through the Middle East to Mongolia and western/northern China, squamous cell carcinoma is the dominant histologic subtype, with incidence declining over the past decades. ${ }^{3}$ The main risk factors for squamous cell carcinoma of the esophagus include smoking and alcohol consumption; other risk factors may include drinking hot beverages and limited inclusion of fruits and vegetables in the diet. ${ }^{3}$ Recent genomic analysis has further shown the differences between histologic subtypes 
of esophageal carcinoma. Adenocarcinomas are fairly homogeneous and are distinct from squamous cell carcinomas, which include at least three molecular subtypes. ${ }^{4}$

No chemotherapeutic regimens or targeted agents are approved specifically for the esophageal carcinoma indication. More than half of patients with esophageal carcinoma present with advanced or metastatic disease, and treatment is largely palliative, is associated with substantial toxicity, and has limited survival benefit. ${ }^{5,6}$ There are few randomized clinical trials in squamous cell carcinoma or adenocarcinoma of the esophagus specifically, and clinicians usually extrapolate evidence from clinical trials in gastric cancer or gastroesophageal junction (GEJ) adenocarcinoma to esophageal carcinoma. For first-line treatment of patients with squamous cell carcinoma or adenocarcinoma of the esophagus, guidelines from the National Comprehensive Cancer Network recommend chemotherapy with cisplatin or oxaliplatin combined with fluorouracil or capecitabine. ${ }^{7}$ Although addition of epirubicin, irinotecan, and taxanes can offer some additional benefit, these combination chemotherapy regimens result in overall response rates (ORRs) $<50 \%$, median overall survival (OS) $<11$ months, and substantial toxicity. ${ }^{7,8}$ Depending on prior therapy and Eastern Cooperative Oncology Group (ECOG) performance status, National Comprehensive Cancer Network guidelines recommend the vascular endothelial growth factor receptor- 2 monoclonal antibody ramucirumab in combination with paclitaxel or single-agent chemotherapy with docetaxel or irinotecan as second-line treatment of advanced or metastatic disease; however, survival benefits with these agents are modest. ${ }^{7}$ Regardless of histologic subtype, patients with advanced or metastatic esophageal carcinoma have a poor prognosis, with a 5 -year OS rate $<15 \%^{5,6}$; therefore, there is a high unmet medical need for therapies that improve outcomes for these patients.

During normal functioning of the immune system, the programmed death-1 (PD-1) receptor is expressed on activated $\mathrm{T}$ cells and interacts with its ligands, programmed death ligand-1 (PD-L1) and programmed death ligand-2, to protect healthy cells from excessive inflammatory or autoimmune responses.9-13 Tumor-associated dysregulation of the PD-1 pathway leads to escape from immune surveillance because tumor cells expressing PD-L1 can reduce T-cell effector activity and dampen appropriate immune responses. ${ }^{13-15}$ In multiple cancer types, host tumorinfiltrating lymphocytes (TILs) mediate PD-L1 expression via interferon- $\gamma$ secretion. ${ }^{13,14}$ PD-L1 overexpression has been observed in up to $40 \%$ of squamous cell carcinomas of the esophagus and in up to $18 \%$ of esophageal adenocarcinomas, and although prognosis is worse for patients with squamous cell carcinoma overexpressing PD-L1 than for those without PD-L1 expression, the prognostic impact of PD-L1 expression in esophageal adenocarcinoma is not well understood. ${ }^{16,17}$ Furthermore, TIL density correlates with therapeutic tumor response and survival in esophageal carcinoma, and TIL composition of the tumor is inversely correlated with expression levels of negative immune regulators. ${ }^{18-21}$ Taken together, the immune checkpoint dysregulation and inflamed phenotype demonstrated in multiple tumor types provides a compelling rationale for evaluation of immune checkpoint inhibitors in solid tumors, including esophageal carcinoma.

Pembrolizumab is a high-affinity, humanized immunoglobulin G4-K monoclonal antibody against PD-1 that directly blocks the interaction between PD-1 and its ligands. Pembrolizumab has demonstrated robust antitumor activity and a favorable safety profile in multiple tumor types and is currently approved in more than 60 countries for the treatment of one or more advanced malignancies. In addition, PD-L1 expression has been correlated with a higher treatment response to PD-1 blockade in several different cancer types. ${ }^{22}$ Furthermore, immune-related gene expression signatures, including genes of T-cell function, antigen presentation, and interferon- $\gamma$ signaling, may be strong predictors of clinical benefit from pembrolizumab treatment, even among PD-L1-positive patients. ${ }^{23}$ Although rare in esophageal carcinoma, microsatellite instability-high status and presence of Epstein-Barr virus infection may also affect response to anti-PD-1 therapy. ${ }^{24}$ Herein, we report the safety, efficacy, and gene expression signature score of a cohort of patients with recurrent or metastatic PDL1-positive carcinoma of the esophagus or GEJ who were treated with pembrolizumab in the multicohort KEYNOTE-028 study.

\section{PATIENTS AND METHODS}

\section{Study Design and Patients}

KEYNOTE-028 (ClinicalTrials.gov identifier: NCT02054806) is an international, multicenter, multicohort, phase IB trial of pembrolizumab in patients with 20 different types of PD-L1-positive advanced solid tumors with high unmet need. Patients in the esophageal carcinoma cohort were enrolled at nine investigational sites in France, Japan, the Republic of Korea, Taiwan, the United Kingdom, and the United States.

Key eligibility criteria for this cohort included age $\geq 18$ years; measurable disease at baseline on the basis of Response Evaluation Criteria in Solid Tumors, version 1.1 (RECIST v 1.1); ECOG performance status of 0 or 1 ; adequate organ (coagulation, hematologic, hepatic, and renal) function as determined by laboratory tests within 10 days of study treatment; provision of archival tumor tissue for gene expression profiling; and PD-L1-positive, histologically or cytologically confirmed, locally advanced, or metastatic squamous cell carcinoma or adenocarcinoma of the esophagus or GEJ for which prior standard therapy was ineffective or for which standard therapy did not exist or was not considered appropriate.

Key exclusion criteria were diagnosis of immunodeficiency or receipt of systemic steroid therapy within 7 days before the first pembrolizumab dose; treatment with any anticancer monoclonal antibody within 4 weeks before the first pembrolizumab dose; prior chemotherapy, small-molecule therapy, or radiation therapy within 2 weeks before the first pembrolizumab dose; active brain metastases; active autoimmune disease that necessitated systemic treatment in the preceding 2 years; interstitial lung disease; previous therapy targeting T-cell costimulation or checkpoint pathways; and human immunodeficiency virus, hepatitis B, or hepatitis C infection.

\section{Treatment and Assessments}

Patients received pembrolizumab intravenously at a dose of $10 \mathrm{mg} / \mathrm{kg}$ once every 2 weeks for up to 2 years or until confirmed progression, intolerable toxicity, or patient or investigator decision to discontinue. Computed tomography or magnetic resonance imaging was performed every 8 weeks for the first 6 months and every 12 weeks thereafter. Response was assessed per RECIST v1.1, as assessed by investigator review. Adverse events (AEs) were reported throughout treatment and for 30 days after discontinuation ( 90 days for serious AEs) and were graded according to the National Cancer Institute Common Terminology Criteria for Adverse Events, version 4.0. AEs of special interest were defined as events with potentially drug-related immunologic causes that were consistent with an immune phenomenon, regardless of attribution to treatment or immune relatedness by the investigator. The primary end points were safety and ORR by investigator review. ORR was defined as the proportion of patients who experienced complete response or partial response on the basis of 


\begin{tabular}{|c|c|}
\hline Characteristic & No. (\%) \\
\hline Median age, years (range) & $65(26-71)$ \\
\hline \multicolumn{2}{|l|}{ Sex } \\
\hline Male & $19(83)$ \\
\hline Female & $4(17)$ \\
\hline \multicolumn{2}{|l|}{ Race } \\
\hline Asian & $12(52)$ \\
\hline White & $7(30)$ \\
\hline Black or African American & $1(4)$ \\
\hline Not specified & $3(13)$ \\
\hline \multicolumn{2}{|l|}{ ECOG performance status } \\
\hline 0 & $8(35)$ \\
\hline 1 & $15(65)$ \\
\hline \multicolumn{2}{|l|}{ Baseline lactate dehydrogenase level } \\
\hline Normal & $17(74)$ \\
\hline Elevated & $6(26)$ \\
\hline \multicolumn{2}{|l|}{ Histology at baseline } \\
\hline Squamous cell carcinoma & $18(78)$ \\
\hline Adenocarcinoma & $5(22)$ \\
\hline Prior radiation therapy & $14(61)$ \\
\hline Prior (neo)adjuvant systemic therap & $6(26)$ \\
\hline \multicolumn{2}{|c|}{ Prior lines of therapy for advanced/metastatic disease } \\
\hline 0 & $1(4)^{*}$ \\
\hline 1 & $2(9)$ \\
\hline 2 & $9(39)$ \\
\hline$\geq 3$ & $11(48)$ \\
\hline \multicolumn{2}{|l|}{ Type of prior therapyt } \\
\hline Platinum & $23(100)$ \\
\hline Fluoropyrimidine & $21(91)$ \\
\hline Taxane & $17(74)$ \\
\hline Epirubicin & $3(13)$ \\
\hline Irinotecan & $3(13)$ \\
\hline Trastuzumab & $1(4)$ \\
\hline \multicolumn{2}{|c|}{$\begin{array}{l}\text { Abbreviation: ECOG, Eastern Cooperative Oncology Group. } \\
\text { *This patient received prior (neo)adjuvant therapy with oxaliplatin, fluorouracil, } \\
\text { and levoleucovorin calcium for advanced/metastatic disease. } \\
\text { tlncludes therapy given in the (neo)adjuvant setting. Patients could have re- } \\
\text { ceived } \geq 1 \text { type of prior therapy. Not all prior therapies are listed. }\end{array}$} \\
\hline
\end{tabular}

RECIST v1.1 at any time during the study; a confirmatory assessment of response was required per RECIST v1.1. Secondary end points were progression-free survival (PFS), defined as time from enrollment to first documented disease progression according to RECIST v1.1 or death from any cause; OS, defined as time from enrollment to death from any cause; and duration of response (DOR), defined as time from first response to disease progression in patients who achieved partial response or better.

\section{PD-L 1 Expression Analysis}

PD-L1 expression was assessed at screening by a central laboratory on the basis of a laboratory-developed prototype immunohistochemical assay (QualTek Molecular Laboratories, Goleta, CA) using either an archived (formalin-fixed, paraffin-embedded) tumor sample or newly obtained biopsy sample ${ }^{23}$ and the 22C3 antibody (Merck, Kenilworth, NJ). PD-L1 positivity was defined as membranous staining on at least $1 \%$ of scorable cells or the presence of a distinctive interface pattern in both neoplastic cells and contiguous mononuclear inflammatory cells. ${ }^{25}$

\section{Gene Expression Profiling}

Tumor tissue was collected at baseline before treatment of a six-gene interferon- $\gamma$ gene expression signature (CXCL9, CXCL10, HLA-DRA, IDO1, IFNG, and STAT1) previously found to be associated with response to pembrolizumab in a melanoma test $\operatorname{set}^{26}$ and subsequently confirmed in head and neck ${ }^{27}$ and gastric ${ }^{23}$ cancer test sets. Samples consisted of formalin-fixed, paraffin-embedded blocks and/or cut tissue on slides. Total RNA was isolated from samples using the RecoverAll kit (Thermo Fisher

\begin{tabular}{|c|c|c|}
\hline $\mathrm{AE}$ & $\begin{array}{c}\text { Grade } 1 \text { or } 2 \\
\text { No. }(\%)\end{array}$ & $\begin{array}{l}\text { Grade } 3 \\
\text { No. }(\%)^{*}\end{array}$ \\
\hline Asthenia & $1(4)$ & 0 \\
\hline Decreased appetite & $1(4)$ & $1(4)$ \\
\hline Decreased lymphocyte count & 0 & $2(9)$ \\
\hline Decreased WBC count & $1(4)$ & 0 \\
\hline Dehydration & $1(4)$ & 0 \\
\hline Dental caries & $1(4)$ & 0 \\
\hline Fatigue & $1(4)$ & 0 \\
\hline Hyperthyroidism & $1(4)$ & 0 \\
\hline Hypothyroidism & $1(4)$ & 0 \\
\hline Increased lacrimation & $1(4)$ & 0 \\
\hline Liver disorder & 0 & $1(4)$ \\
\hline Pemphigoid & $1(4)$ & 0 \\
\hline Pneumonia & $1(4)$ & 0 \\
\hline Pruritus & $1(4)$ & 0 \\
\hline Rash & $2(9) \dagger$ & $1(4) \neq$ \\
\hline Stomatitis & $1(4)$ & 0 \\
\hline \multicolumn{3}{|c|}{$\begin{array}{l}\text { Abbreviation: AE, adverse event. } \\
\text { *There were no grade } 4 \text { treatment-related AEs. } \\
\text { tIncludes rash and generalized rash. } \\
\text { ‡Generalized rash. }\end{array}$} \\
\hline
\end{tabular}

Scientific, Austin, TX), and RNA was quantified using the NanoDrop ND1000 spectrophotometer (Thermo Fisher Scientific, Wilmington, $\mathrm{DE})$. The six-gene interferon- $\gamma$ gene expression signature was calculated as the arithmetic average of the log ratio of the counts of the predictor genes to the geometric mean of the counts of a set of 11 housekeeping normalization genes. Tests of association between the interferon- $\gamma$ signature score and PFS and ORR were based on Cox and logistic regression modeling.

\section{Study Oversight}

The study protocol (No. MK-3475-028-02) and all amendments were approved by the appropriate institutional review boards and ethics committees at each participating institution. The study was conducted in accordance with the protocol, Good Clinical Practice guidelines, and the ethical principles outlined in the Declaration of Helsinki. All patients provided written informed consent to participate.

\section{Statistical Analyses}

Per protocol, multiple interim analyses could be performed because of the sequential design of the study. A sequential monitoring procedure was used to evaluate efficacy and futility after at least six patients had at least one postbaseline response assessment. Enrollment continued provided at least one of the first six patients responded. A sample size of 22 evaluable patients in this cohort was calculated, using the binomial exact method, to provide $80 \%$ power to demonstrate that the best ORR exceeded $10 \%$ at an overall one-sided $8 \% \alpha$ level if the true ORR was 35\%. The efficacy analysis population included all patients with measurable disease at

Table 3. Best Overall Response per Response Evaluation Criteria in Solid Tumors, Version 1.1, Assessed by Investigator Review $(\mathrm{N}=23)$

\begin{tabular}{lrrr}
\hline \multicolumn{1}{c}{ Response } & No. & $\%$ & $95 \% \mathrm{Cl}$ \\
\hline Overall response rate & 7 & 30 & 13 to 53 \\
Complete response & 0 & 0 & 0 to 15 \\
Partial response & 7 & 30 & 13 to 53 \\
Stable disease & 2 & 9 & 1 to 28 \\
Progressive disease & 13 & 57 & 35 to 77 \\
Nonevaluable & 1 & 4 & $<1$ to 22 \\
\hline
\end{tabular}


baseline (according to RECIST v1.1) who received at least one dose of pembrolizumab. The safety analysis population included all patients who received at least one dose of pembrolizumab. The truncated sequential probability test was used to evaluate ORR. The Kaplan-Meier method was used to estimate PFS, OS, and DOR. SAS, version 9.3 (SAS Institute, Cary, $\mathrm{NC}$ ), or R, version 3.1.3, was used for all analyses. The data cutoff date for all analyses presented herein was February 20, 2017.

\section{RESULTS}

\section{PD-L1 Expression and Baseline Characteristics}

Samples were evaluable for assessment of tumor PD-L1 expression in 83 of the 90 patients screened (Appendix Fig A1, online only). Thirty seven $(45 \%)$ of these samples were determined to be PD-L1-positive using a prototype immunohistochemical assay. Among the patients with PD-L1-positive tumors, 14 did not meet the inclusion criteria for the trial, most commonly because they declined study participation $(\mathrm{n}=6)$ or did not have an ECOG performance status of 0 or $1(n=5)$. The other 23 patients were enrolled from March to July 2014.

Median age was 65 years, $83 \%$ of patients were men, and $78 \%$ had squamous cell carcinoma histology (Table 1). More than half the patients $(61 \%)$ had received previous radiation therapy. All but one patient had received previous therapy for advanced/metastatic disease; $87 \%$ had received at least two previous therapies for advanced/metastatic disease, and all patients had received platinumbased therapy for early-stage or advanced/metastatic disease. As of the data cutoff date, median follow-up duration was 7 months (range, 1 to 33 months) and 21 patients (91\%) had discontinued treatment, most commonly for progressive disease (57\%) and AEs (9\%).

\section{Safety}

All 23 patients were included in the safety analysis population. All-grade treatment-related AEs occurred in nine patients

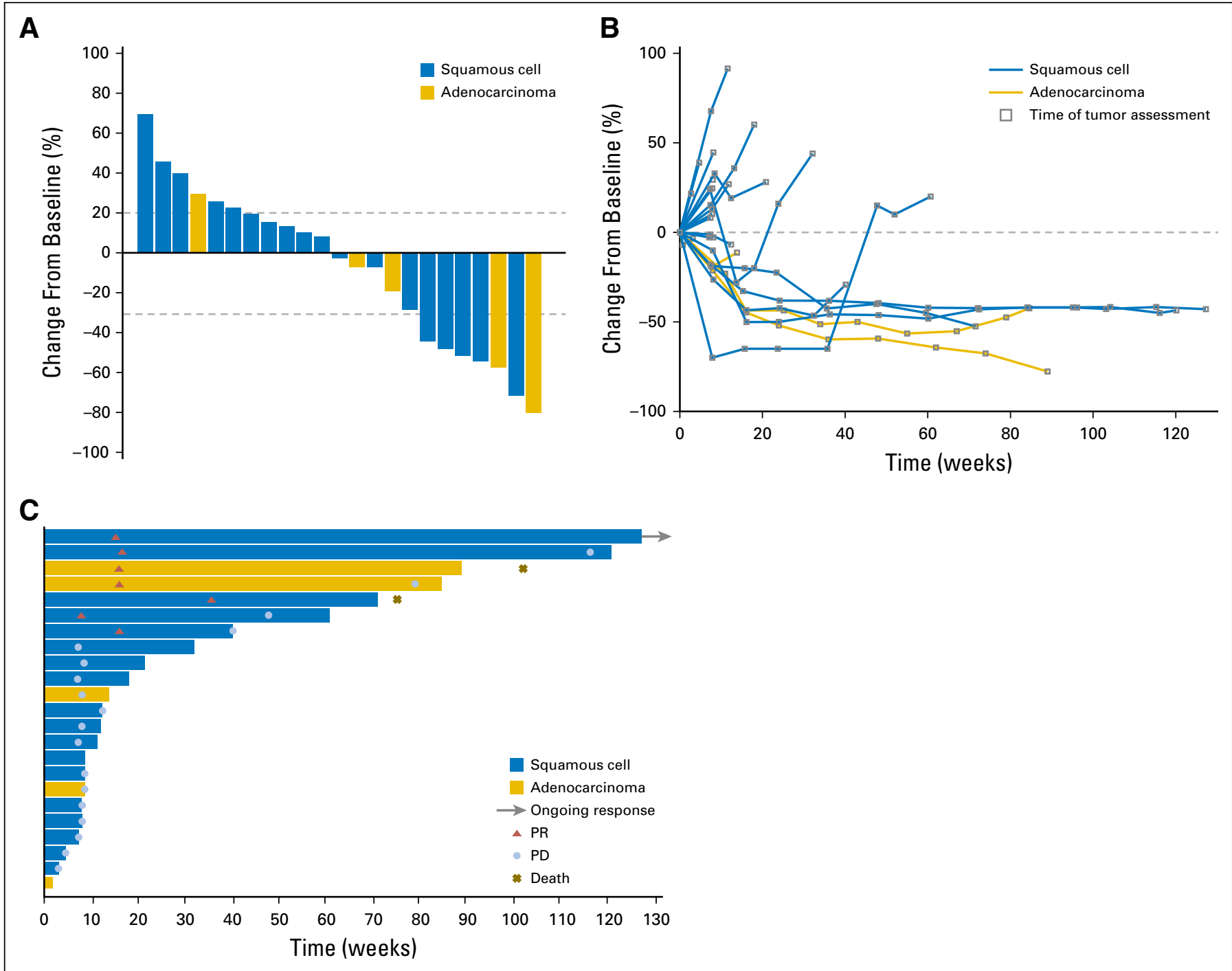

Fig 1. (A) Best percentage change from baseline in tumor size per Response Evaluation Criteria in Solid Tumors, version 1.1 (RECIST v1.1) by investigator review. The

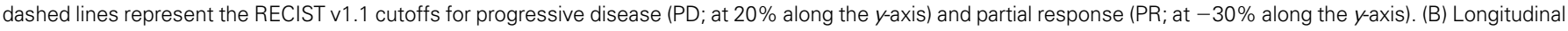

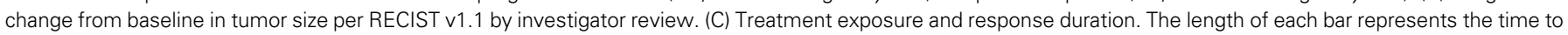
the last radiographic assessment. 
(39\%; Table 2), most commonly rash/generalized rash in three patients $(13 \%)$ and decreased appetite and decreased lymphocyte count in two patients $(9 \%)$ each. Grade 3 treatment-related AEs occurred in four patients (17\%; Table 2) and included decreased lymphocyte count in two patients $(9 \%)$ and decreased appetite, liver disorder, and generalized rash in one patient $(4 \%)$ each. All of the grade 3 treatment-related AEs resolved with either discontinuation (for liver disorder), no action taken (for decreased appetite and one event of decreased lymphocyte count), or dose interruption (for one event of decreased lymphocyte count and for generalized rash). The grade 3 treatment-related $\mathrm{AE}$ of generalized rash was considered a prespecified immune-related $\mathrm{AE}$ and occurred on day 19 after initiation of pembrolizumab treatment. There were no grade 4 treatment-related AEs. Treatment-related serious AEs occurred in three patients (13\%), namely, grade 2 pemphigoid, grade 3 decreased appetite, and grade 3 liver disorder in one patient each; all resolved with either discontinuation (for liver disorder) or no action taken (for pemphigoid and decreased appetite). No patients died because of a treatment-related AE. AEs of special interest, regardless of attribution to treatment or immune relatedness by the investigator, occurred in six patients $(26 \%)$ and included hypothyroidism in two patients (9\%) and adrenal insufficiency, enterocolitis, hyperthyroidism, and generalized rash (as described previously) in one patient (4\%) each.

\section{Antitumor Activity}

All 23 patients were included in the efficacy analysis population. On the basis of RECIST v1.1 by investigator review, ORR was $30 \%$ (95\% CI, $13 \%$ to 53\%; Table 3). All seven responses were confirmed partial responses. Among the seven patients with a confirmed partial response, three $(43 \%)$ had received prior radiation therapy. There was no discernible pattern with regard to previous chemotherapy regimen (type, time, or schedule) among the seven patients with a confirmed partial response. Two additional patients experienced confirmed stable disease. Responses were observed in patients with both squamous and adenocarcinoma histology. By histologic subtype, ORR was $28 \%$ (five of 18 patients) for patients with squamous cell carcinoma and $40 \%$ (two of five patients) for those with adenocarcinoma. Twelve patients (52\%) experienced a decrease from baseline in target lesion burden (Fig 1A), which was generally maintained across subsequent tumor assessments (Fig 1B). The median decrease from baseline in target lesion burden for the 12 patients who experienced tumor shrinkage was $-44.7 \%$ (range, $-77.7 \%$ to $-2.7 \%$ ).

For the seven patients with a partial response, determined by investigator review, median time to initial response was 4 months (range, 2 to 8 months) and median DOR was 15 months (range, 6 to $\geq 26$ months). Patients who experienced a partial response most commonly had target lesions in the lungs $(n=4)$, lymph nodes $(n=3)$, and liver $(n=2)$. At the time of analysis, one of the seven partial responses was ongoing, but all of the patients had discontinued treatment (Fig 1C).

On the basis of investigator review, median PFS was 1.8 months (95\% CI, 1.7 to 2.9 months), and the 6-month and 12-month PFS rates were $30 \%$ and $22 \%$, respectively (Fig 2A). Median OS was 7.0 months (95\% CI, 4.3 to 17.7 months), and the 6-month and 12-month OS rates were $60 \%$ and $40 \%$, respectively (Fig 2B).

The pattern of PFS according to the six-gene interferon- $\gamma$ gene expression signature score is shown in Figure 3. The signature score showed trends toward an association with PFS $(P=.053$, onesided) and ORR $(P=.107$, one-sided $)$ in patients treated with pembrolizumab.

\section{DISCUSSION}

There is a high unmet need for effective and well-tolerated treatments for patients with advanced esophageal carcinoma. The overall safety profile in this study of patients with recurrent or metastatic PD-L1-positive esophageal carcinoma was similar to that previously reported for pembrolizumab in patients with other advanced malignancies, ${ }^{22,23,25-29}$ and no unexpected treatmentrelated AEs occurred. Because the anti-PD-1 antibody pembrolizumab targets immune cells and not tumor cells, and because of the activity of pembrolizumab monotherapy previously observed in patients with both squamous cell and adenocarcinoma histology in other tumor types, including gastric, head and neck,
A

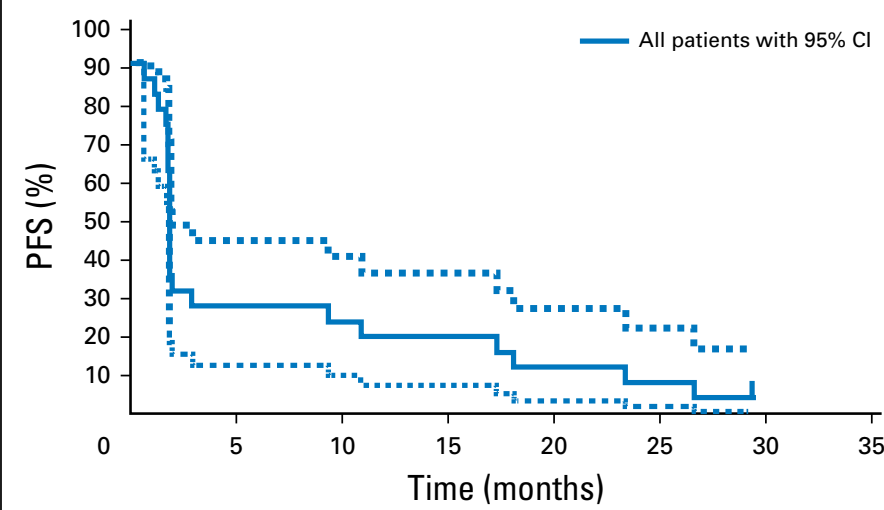

No. at risk:

23

$7 \quad 6$

5

3

2

0

0

B

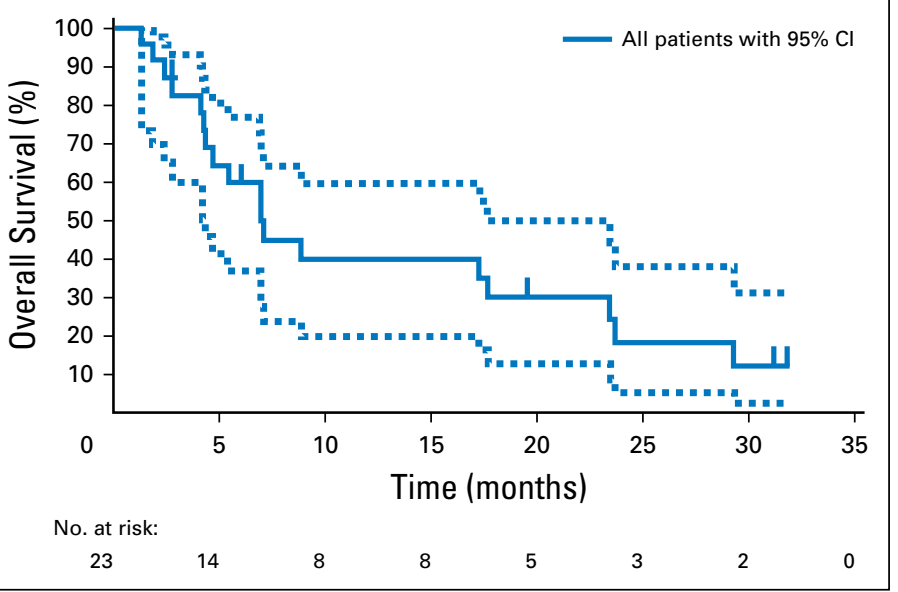

Fig 2. (A) Progression-free survival (PFS) per Response Evaluation Criteria in Solid Tumors, version 1.1 (RECIST v1.1) by investigator review and (B) overall survival. 


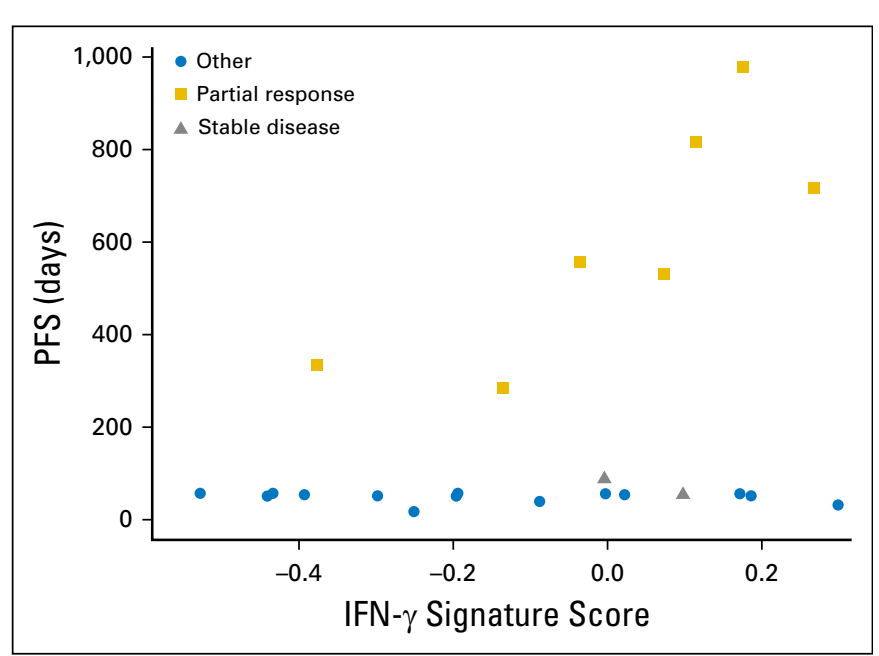

Fig 3. Interferon (IFN)- $\gamma$ gene signature scores and association with progressionfree survival (PFS).

and non-small-cell lung cancer, ${ }^{23,27,29}$ we expected antitumor activity in esophageal cancer regardless of histologic subtype; therefore, patients with both squamous cell carcinoma and adenocarcinoma (including GEJ) of the esophagus were enrolled in this cohort. Pembrolizumab was associated with promising preliminary antitumor activity and manageable toxicity in patients with PD-L1-positive, heavily pretreated, advanced esophageal carcinoma. Overall, tumor shrinkage from baseline in target lesions occurred in more than half the patients. Similar to other tumor types, the extent of response to pembrolizumab varied by histologic subtype, with $40 \%$ of patients with adenocarcinoma and $28 \%$ of patients with squamous cell carcinoma experiencing a partial response, for an overall ORR of 30\% (95\% CI, 13\% to $53 \%)$. Responses occurred early, with a median time to initial response of 4 months (range, 2 to 8 months), and were durable, with a median DOR of 15 months (range, 6 to $\geq 26$ months). Even patients with stable disease experienced initial tumor shrinkage at first radiologic assessment, which was not indicative of pseudoprogression. Among those patients who did have progressive disease, they were mainly cases of primary resistance. Because of the small number of patients with adenocarcinoma, conclusions regarding PFS or OS by histologic subtype are not possible. Antitumor activity of the anti-PD-1 antibody nivolumab was also recently shown in a phase II study in which $17 \%$ of patients ( $\mathrm{n}=65$ ) with PD-L1 unselected treatment-refractory squamous cell carcinoma of the esophagus had a centrally assessed objective response. $^{30}$

On the basis of evidence of the involvement of the PD-1/PD-L1 pathway in esophageal carcinoma, ${ }^{16,18}$ tumor PD-L1 positivity was an enrollment criterion in this study. Among patients screened for enrollment, $45 \%$ had PD-L1-positive tumors as determined by a prototype immunohistochemistry assay, supporting a role for this pathway in at least a subset of esophageal carcinomas. Similar to results from other studies of immune checkpoint inhibition in various tumor types, ${ }^{22,23,29}$ only a subset of patients with PDL1-positive esophageal cancer showed clinical benefit from PD-1 blockade in this study, suggesting that additional biomarkers may be necessary for reliable prediction of clinical response to pembrolizumab. On the basis of a six-gene interferon- $\gamma$ gene expression signature analysis of CXCL9, CXCL10, HLA-DRA, IDO1, IFNG, and STAT1, delays in progression and increased clinical response tended to occur among pembrolizumab-treated patients with esophageal carcinoma and higher signature scores, consistent with findings published for head and neck and gastric cancer. ${ }^{23,27}$ However, there were limitations to this analysis, including a small sample size, variability in tissue biopsy storing conditions, and potential sampling bias and heterogeneity. These preliminary results regarding the potential predictive value of gene expression signature for pembrolizumab activity in esophageal carcinoma will therefore require additional exploration in a larger patient population. In addition, because all patients enrolled in KEYNOTE028 had tumors with some degree of PD-L1 expression (as demonstrated by the prototype assay), this study cannot provide information on the potential activity of pembrolizumab in patients with advanced esophageal carcinoma and truly PD-L1-negative tumors.

In conclusion, pembrolizumab demonstrated promising preliminary antitumor activity and a manageable safety profile in this heavily pretreated population of patients with PD-L1-positive advanced squamous cell carcinoma or adenocarcinoma of the esophagus enrolled in KEYNOTE-028. Evaluation of pembrolizumab for the treatment of esophageal carcinoma is ongoing.

\section{AUTHORS' DISCLOSURES OF POTENTIAL CONFLICTS OF INTEREST}

Disclosures provided by the authors are available with this article at jco.org.

\section{AUTHOR CONTRIBUTIONS}

Provision of study materials or patients: Sarina A. Piha-Paul Collection and assembly of data: Toshihiko Doi, Sarina A. Piha-Paul, Shadia I. Jalal, Minori Koshiji

Data analysis and interpretation: Shadia I. Jalal, Sanatan Saraf, Jared Lunceford, Minori Koshiji, Jaafar Bennouna

Manuscript writing: All authors

Final approval of manuscript: All authors

Accountable for all aspects of the work: All authors

\section{REFERENCES}

1. Jemal A, Bray F, Center MM, et al: Global cancer statistics. CA Cancer J Clin 61:69-90, 2011

2. Ferlay J, Soerjomataram I, Dikshit R, et al: Cancer incidence and mortality worldwide: Sources, methods and major patterns in GLOBOCAN 2012. Int J Cancer 136:E359-E386, 2015

3. Zhang HZ, Jin GF, Shen HB: Epidemiologic differences in esophageal cancer between Asian and Western populations. Chin J Cancer 31:281-286, 2012

4. Integrated genomic characterization of oesophageal carcinoma. Nature 541:169-175, 2017
5. Tew WP, Kelsen DP, Ilson DH: Targeted therapies for esophageal cancer. Oncologist 10:590-601, 2005

6. D'Journo XB, Thomas PA: Current management of esophageal cancer. J Thorac Dis 6:S253-S264, 2014 (suppl 2)

7. National Comprehensive Cancer Network: NCCN clinical practice guidelines in oncology. Esophageal 
and esophagogastric junction cancers v.2 2016. https://www.nccn.org/professionals/physician_gls/ pdf/esophageal.pdf

8. Wiedmann MW, Mössner J: New and emerging combination therapies for esophageal cancer. Cancer Manag Res 5:133-146, 2013

9. Keir ME, Butte MJ, Freeman GJ, et al: PD-1 and its ligands in tolerance and immunity. Annu Rev Immunol 26:677-704, 2008

10. Carter L, Fouser LA, Jussif J, et al: PD-1:PD-L inhibitory pathway affects both $\mathrm{CD} 4(+)$ and $\mathrm{CD} 8(+)$ $\mathrm{T}$ cells and is overcome by IL-2. Eur $\mathrm{J}$ Immunol 32: 634-643, 2002

11. Freeman GJ, Long AJ, Iwai $Y$, et al: Engagement of the PD-1 immunoinhibitory receptor by a novel B7 family member leads to negative regulation of lymphocyte activation. J Exp Med 192: 1027-1034, 2000

12. Latchman $Y$, Wood $C R$, Chernova $T$, et al: PD$\mathrm{L} 2$ is a second ligand for PD-1 and inhibits $T$ cell activation. Nat Immunol 2:261-268, 2001

13. McDermott DF, Atkins MB: PD-1 as a potential target in cancer therapy. Cancer Med 2:662-673, 2013

14. Pardoll DM: The blockade of immune checkpoints in cancer immunotherapy. Nat Rev Cancer 12: 252-264, 2012

15. He J, Hu Y, Hu M, et al: Development of PD-1/ PD-L1 pathway in tumor immune microenvironment and treatment for non-small cell lung cancer. Sci Rep 5:13110, 2015
16. Ohigashi $Y$, Sho $M$, Yamada $Y$, et al: Clinical significance of programmed death-1 ligand-1 and programmed death-1 ligand-2 expression in human esophageal cancer. Clin Cancer Res 11:2947-2953, 2005

17. Derks S, Nason KS, Liao X, et al: Epithelial PDL2 expression marks Barrett's esophagus and esophageal adenocarcinoma. Cancer Immunol Res 3: 1123-1129, 2015

18. Lu B, Chen L, Liu L, et al: T-cell-mediated tumor immune surveillance and expression of $B 7$ co-inhibitory molecules in cancers of the upper gastrointestinal tract. Immunol Res 50:269-275, 2011

19. Lv L, Pan K, Li XD, et al: The accumulation and prognosis value of tumor infiltrating $\mathrm{IL}-17$ producing cells in esophageal squamous cell carcinoma. PLoS One 6:e18219, 2011

20. Schumacher K, Haensch W, Röefzaad C, et al: Prognostic significance of activated CD8(+) T cell infiltrations within esophageal carcinomas. Cancer Res 61:3932-3936, 2001

21. Cho $Y$, Miyamoto $M$, Kato $K$, et al: $C D 4^{+}$and $\mathrm{CD}^{+} \mathrm{T}$ cells cooperate to improve prognosis of patients with esophageal squamous cell carcinoma. Cancer Res 63:1555-1559, 2003

22. Topalian SL, Hodi FS, Brahmer JR, et al: Safety, activity, and immune correlates of anti-PD-1 antibody in cancer. N Engl J Med 366:2443-2454, 2012

23. Muro K, Chung HC, Shankaran V, et al: Pembrolizumab for patients with PD-L1-positive advanced gastric cancer (KEYNOTE-012): A multicentre, openlabel, phase 1b trial. Lancet Oncol 17:717-726, 2016

24. Jin Z, Yoon HH: The promise of PD-1 inhibitors in gastro-esophageal cancers: Microsatellite instability vs. PD-L1. J Gastrointest Oncol 7:771-788, 2016

25. Dolled-Filhart M, Locke D, Murphy $T$, et al: Development of a prototype immunohistochemistry assay to measure programmed death ligand-1 expression in tumor tissue. Arch Pathol Lab Med 140: 1259-1266, 2016

26. Ribas $A$, Robert $C$, Hodi $S$, et al: Response to programmed death receptor 1 (PD-1) blockade with pembrolizumab (MK-3475) is associated with an interferon-inflammatory immune gene signature. J Clin Oncol 33, 2015 (suppl; abstr 3001)

27. Seiwert TY, Burtness B, Mehra R, et al: Safety and clinical activity of pembrolizumab for treatment of recurrent or metastatic squamous cell carcinoma of the head and neck (KEYNOTE-012): An open-label, multicentre, phase 1b trial. Lancet Oncol 17:956-965, 2016

28. Robert C, Schachter J, Long GV, et al: Pembrolizumab versus ipilimumab in advanced melanoma. N Engl J Med 372:2521-2532, 2015

29. Garon EB, Rizvi NA, Hui $R$, et al: Pembrolizumab for the treatment of non-small-cell lung cancer. N Engl J Med 372:2018-2028, 2015

30. Kudo T, Hamamoto $Y$, Kato K, et al: Nivolumab treatment for oesophageal squamous-cell carcinoma: An open-label, multicentre, phase 2 trial. Lancet Oncol 18:631-639, 2017

\section{Affiliations}

Toshihiko Doi, National Cancer Center East, Chiba, Japan; Sarina A. Piha-Paul, The University of Texas MD Anderson Cancer Center, Houston, TX; Shadia I. Jalal, Indiana University School of Medicine, Indianapolis, IN; Sanatan Saraf, Jared Lunceford, and Minori Koshiji, Merck, Kenilworth, NJ; and Jaafar Bennouna, Institut de Cancérologie de l'Ouest, Nantes, France.

Supported by Merck, Kenilworth, NJ.

\section{Support}

\section{Prior Presentation}

Presented in part at the American Society of Clinical Oncology Annual Meeting, Chicago, IL, May 29-June 2, 2015; Gastrointestinal Cancers Symposium, San Francisco, CA, January 21-23, 2016; and American Society of Clinical Oncology Annual Meeting, Chicago, IL, June 3-7, 2016.

\section{Conquer Cancer Foundation of the American Society of Clinical Oncology}

The Conquer Cancer Foundation (CCF), created by the world's foremost cancer physicians of the American Society of Clinical Oncology, seeks dramatic advances in the prevention, treatment, and cures of all types of cancer. Working toward the vision of a world free from the fear of cancer, CCF invests in doctors and scientists at every stage of their career by funding breakthrough cancer research, sharing cutting-edge knowledge, and improving the quality of and access to care. For more information, visit CONQUER.ORG. 
Safety and Antitumor Activity of the Anti-Programmed Death-1 Antibody Pembrolizumab in Patients With Advanced Esophageal Carcinoma

The following represents disclosure information provided by authors of this manuscript. All relationships are considered compensated. Relationships are self-held unless noted. I = Immediate Family Member, Inst = My Institution. Relationships may not relate to the subject matter of this manuscript. For more information about ASCO's conflict of interest policy, please refer to www.asco.org/rwc or ascopubs.org/jco/site/ifc.

\section{Toshihiko Doi}

Consulting or Advisory Role: Eli Lilly Japan, Chugai Pharma, Kyowa Hakko Kirin, Novartis, MSD, Daiichi Sankyo, Amgen

Research Funding: Taiho Pharmaceutical (Inst), Novartis (Inst), Merck Serono (Inst), Astellas Pharma (Inst), MSD (Inst), Janssen (Inst), Boehringer Ingelheim (Inst), Takeda (Inst), Pfizer (Inst), Eli Lilly Japan (Inst), Sumitomo Group (Inst), Chugai Pharma (Inst), Bayer (Inst), Kyowa Hakko Kirin (Inst), Daiichi Sankyo (Inst), Celgene (Inst)

\section{Sarina A. Piha-Paul}

Consulting or Advisory Role: Genentech

Research Funding: GlaxoSmithKline, Xuan Zhu, Puma Biotechnology, Novartis, Merck Sharp \& Dohme, Curis, Principa Biopharma, Biomarin, Helix BioPharma, Bayer, AbbVie, Incyte, Five Prime Therapeutics, Cerulean Pharma, MedImmune, Medivation

Shadia I. Jalal

Research Funding: AstraZeneca/MedImmune

\section{Sanatan Saraf}

Employment: Merck Sharp \& Dohme

Jared Lunceford

Employment: Merck Sharp \& Dohme

Stock or Other Ownership: Merck Sharp \& Dohme

\section{Minori Koshiji}

Employment: Merck Sharp \& Dohme

Jaafar Bennouna

Honoraria: Roche, Boehringer Ingelheim, AstraZeneca, Shire, Merck Sharp \& Dohme

Consulting or Advisory Role: Roche, Boehringer Ingelheim, AstraZeneca, Shire, Merck Sharp \& Dohme 


\section{Acknowledgment}

The authors thank the patients and their families and caregivers for participating in the study; Mark Ayers, Terri McClanahan, and Michael Nebozhyn (Merck, Kenilworth, NJ) for contributions to the gene expression analysis; Guo Qing Zhao (Merck, Kenilworth, NJ) for statistical support; Roger Dansey (Merck, Kenilworth, NJ) for critical manuscript review; and QualTek Molecular Laboratories (Goleta, CA) for PD-L1 immunohistochemistry assay testing. Medical writing and/or editorial assistance was provided by Sarah Adai and Payal Gandhi of the ApotheCom pembrolizumab team (Yardley, PA). This assistance was funded by Merck, Kenilworth, NJ. 


\section{Appendix}

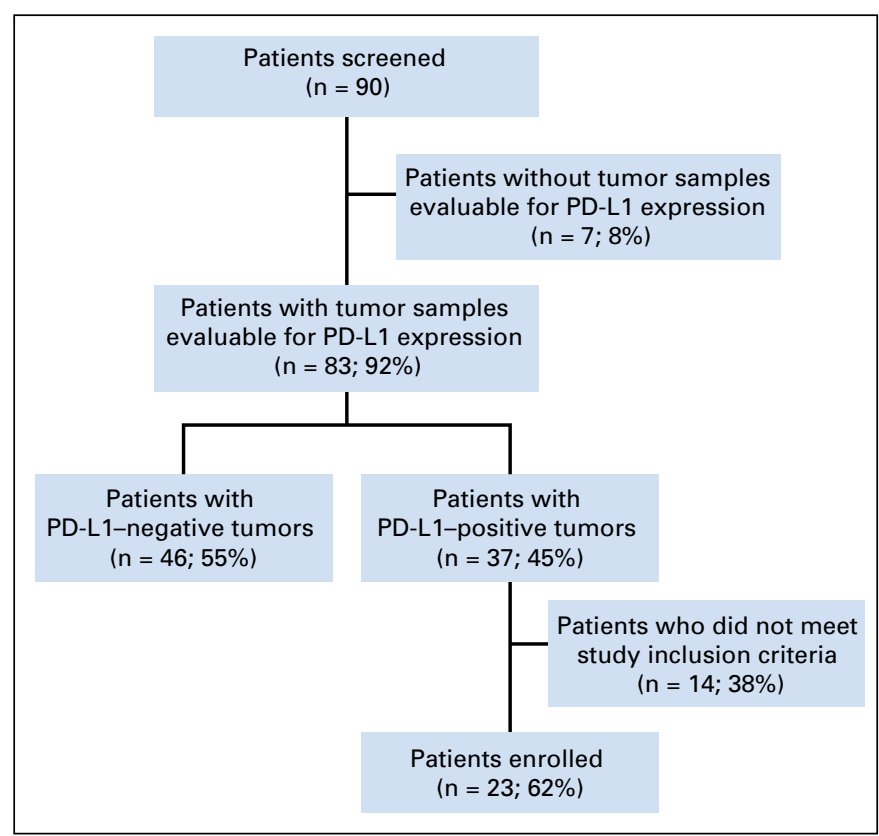

Fig A1. Patient disposition. PD-L1, programmed death ligand 1. 